\title{
LE DROIT DE LA MIGRATION ET SES INTERMÉDIAIRES : USAGES SOCIOPOLITIQUES DU DROIT ET PRODUCTION DES POLITIQUES MIGRATOIRES. PRÉSENTATION DU DOSSIER
}

\author{
Jonathan Miaz, Laura Odasso, Romane Sabrié
}

Lextenso | « Droit et société »

2021/1 N 107 | pages 7 à 15

ISSN 0769-3362

Article disponible en ligne à l'adresse :

https://www.cairn.info/revue-droit-et-societe-2021-1-page-7.htm

Distribution électronique Cairn.info pour Lextenso.

(C) Lextenso. Tous droits réservés pour tous pays.

La reproduction ou représentation de cet article, notamment par photocopie, n'est autorisée que dans les limites des conditions générales d'utilisation du site ou, le cas échéant, des conditions générales de la licence souscrite par votre établissement. Toute autre reproduction ou représentation, en tout ou partie, sous quelque forme et de quelque manière que ce soit, est interdite sauf accord préalable et écrit de l'éditeur, en dehors des cas prévus par la législation en vigueur en France. Il est précisé que son stockage dans une base de données est également interdit. 


\section{Dossier}

Le droit de la migration et ses intermédiaires 


\title{
Le droit de la migration et ses intermédiaires : usages sociopolitiques du droit et production des politiques migratoires. Présentation du dossier
}

\author{
Jonathan Miaz *, Laura Odasso **, Romane Sabrié ***
}

\begin{abstract}
* Centre de droit comparé européen et international (CDCEI), Université de Lausanne, Quartier UNIL-Chamberonne, Bâtiment Internef, Bureau 317, 1004 Lausanne, Suisse. <jonathan.miaz@unil.ch>

** Collège de France, Chaire Migrations et Sociétés ; Institut Convergences Migrations, 3 rue d'Ulm, F-75005 Paris MESOPOLHIS, UMR 7064, Aix-Marseille université-CNRS-Sciences Po Aix. <laura.odasso@college-de-france.fr>

${ }^{* * *}$ Laboratoire de sociologie, philosophie et anthropologie politiques (SOPHIAPOL), Université Paris Nanterre,

200 avenue de la République, F-92001 Nanterre Cedex. <romane.sabrie@gmail.com>
\end{abstract}

En prenant pour objet le droit de la migration et ses intermédiaires, ce dossier éclaire comment les usages du droit d'un éventail hétérogène d'acteurs - administrations, tribunaux, associations, avocat.e.s, militant·e.s - mettent en œuvre ou contestent les politiques migratoires, mais aussi comment les interactions entre leurs différentes pratiques produisent du droit au concret. Ainsi, ce dossier s'inscrit dans la continuité des travaux qui, souvent dans une perspective ethnographique, ont porté sur les politiques migratoires à partir des pratiques de différent.e.s agent.e.s de terrain ${ }^{1}$ au sein des administrations, des tribunaux ou des associations, témoignant de la dimension relationnelle de leur mise en œuvre ${ }^{2}$.

\section{Des acteurs de terrain comme intermédiaires du droit de la migration}

Ces travaux ont d'abord montré que les street-level bureaucrats ${ }^{3}$ qui instruisent les demandes d'asile ou qui décident de l'attribution d'un titre de séjour disposent d'un pouvoir discrétionnaire important. Ce dernier résulte notamment de la marge de manœuvre laissée par les textes de loi quant aux conditions de leur application 4 et de la complexité des situations sociales sur lesquelles les politiques migratoires interviennent ${ }^{5}$. La littérature a également mis en évidence l’importante logique de

1. Nous parlons des agent·e·s de terrain en référence à la littérature sur les street-level organizations, Evelyn Z. BRodKIN et Pierre-Yves BAUdot, "Les agents de terrain, entre politique et action publique ", Sociologies pratiques, 24 (1), 2012, p. 10-18.

2. Voir par exemple les numéros spéciaux suivants : Camille HAMIDI et Mireille PAQUET, « Redessiner les contours de l'État : la mise en œuvre des politiques migratoires », Lien social et Politiques, 83, 2019, p. 5-35 ; Sule TOMKINSON et Jonathan MiAz, "Au cœur des politiques d'asile : perspectives ethnographiques », Politique et Sociétés, 38 (1), 2019, p. 3-18.

3. Michael Lipsky, Street-level Bureaucracy: Dilemmas of the Individual in Public Service, New York: Russell Sage Foundation, 30th ann. Ed., 2010.

4. Sylvain LAURENS, «Les agents de l'État face à leur propre pouvoir. Éléments pour une microanalyse des mots griffonnés en marge des décisions officielles", Genèses, 72, 2008, p. 26-41 ; Alexis SPIRE, "Comment étudier la politique des guichets? Méthodes pour enquêter sur le pouvoir discrétionnaire des agents de l'immigration », Migrations Société, 167 (1), 2017, p. 91-100.

5. Camille HAMIdI et Mireille PAQUET, « Redessiner les contours de l’État... », article cité, p. 8. 
soupçon qui pèse sur les récits des personnes migrantes 6 , en lien avec des habitus bureaucratiques et des savoirs pratiques qui font du doute et de la lutte contre la fraude une part importante de la mission des agent.e.s ${ }^{7}$. Certaines recherches ont aussi montré comment les institutions recrutent et socialisent leurs agent.e.s ${ }^{8}$, et comment elles orientent leurs pratiques décisionnelles à travers des normes secondaires d'application, des instruments d'accélération des procédures et des mécanismes de contrôle des décisions ${ }^{9}$. Ainsi, ces recherches ont déplacé la focale d'analyse sur les politiques migratoires « des discours aux pratiques et des principes juridiques à leur application » 10 , soulignant alors le travail institutionnel de définition et d'interprétation, variables à travers le temps, des catégories de "réfugié·e " et de «migrant.e ", ainsi que de "vrais $v s$ faux réfugiés " 11 . Cette littérature montre que c'est aussi « au niveau de la rue» (at the street-level), ou " sur le terrain », que les conditions d'accès à un droit au séjour se configurent 12 et que les politiques migratoires et le droit en la matière sont produits ${ }^{13}$. Plusieurs auteur.rice.s soulignent également que la gestion de l'immigration et la mise en œuvre du droit en la matière sont un phénomène relationnel et collectif, dans la mesure où, outre les agent·e.s de terrain, une diversité d'acteurs publics ou privés 14 (experts ${ }^{15}$, inter-

6. Didier FASSIN et Carolina Kobelinsky, "Comment on juge l'asile. L'institution comme agent moral ", Revue française de sociologie, 53 (4), 2012, p. 657-688 ; Olga JuBANY, Screening Asylum in a Culture of Disbelief: Truths, Denials and Skeptical Borders, New York: Springer Berlin Heidelberg, 2016 ; Johanna ProbsT, « Entre faits et fiction: l'instruction de la demande d'asile en Allemagne et en France ", Cultures \& Conflits, 84, 2011, p. 63-80.

7. Jill ALPES et Alexis SPIRE, « Dealing with Law in Migration Control: The Powers of Street-level Bureaucrats at French Consulates ", Social \& Legal Studies, 23 (2), 2014, p. 261-274 ; Federica InFANTINO et Andrea REA, «La mobilisation d'un savoir pratique local : Attribution des visas Schengen au consulat général de Belgique à Casablanca », Sociologies pratiques, 24 (1), 2012, p. 67-78.

8. Karen АKОKA et Alexis SPIRE, « Pour une histoire sociale de l'asile politique en France », Pouvoirs, 1 (144), 2013, p. 67-77 ; Lisa Marie BORRELLI, « The Border Inside - Organizational Socialization of Street-level Bureaucrats in the European Migration Regime», Journal of Borderlands Studies, 2019, p. 1-20, Online first <https://doi.org/10.1080/08865655.2019.1676815>.

9. Jonathan MiAz, «Le Droit et ses médiations. Pratiques d'instruction des demandes d'asile et encadrement institutionnel des décisions ", Politique et Sociétés, 38 (1), 2019, p. 71-98.

10. Alexis SPIRE, Accueillir ou reconduire. Enquête sur les guichets de l'immigration, Paris : Raisons d'agir, 2008, p. 8.

11. Karen АкокА, «Distinguer les réfugiés des migrants au $\mathrm{Xx}^{\mathrm{e}}$ siècle : enjeux et usages des politiques de classification ", in Michel AGIER et Anne-Virginie MADEIRA (dir.), Définir les réfugiés, Paris : PUF / Humensis laviedesidees.fr, 2017, p. 47-68.

12. Tobias G. EulE, Inside Immigration Law. Migration Management and Policy Application in Germany, Surrey, Burlington, Ashgate Publishing, Ltd., 2014 ; Carla MASCIA, «How Bureaucracies Shape Access to Rights: The Implementation of Family Reunification in Belgium ", Journal of Ethnic and Migration Studies, 14 février 2020, p. 1-17, on line first <https://doi.org/10.1080/1369183X.2020.1726734>.

13. Federica InFAnTINO, «How Does Policy Change at the Street Level? Local Knowledge, a Community of Practice and EU Visa Policy Implementation in Morocco ", Journal of Ethnic and Migration Studies, 5 septembre 2019, p. 1-19. On line first <https://doi.org/10.1080/1369183X.2019.1662717>.

14. Tobias G. Eule, Lisa Marie Borrelli, Annika LindBERg et Anna WYss, Migrants Before the Law: Contested Migration Control in Europe, Cham : Springer International Publishing, 2019.

15. Anthony GooD, Anthropology and Expertise in the Asylum Courts, Abingdon, Oxon: New York, NY, Routledge-Cavendish, 2007 ; Rosset Damian, " "We Have our own Kitchen" : distance et légitimité dans la production de savoir pour la procédure d'asile », Politique et Sociétés, 38 (1), 2019. 
prètes ${ }^{16}$, entreprises privées ${ }^{17}$ ) peut être impliquée dans le contrôle des frontières et les procédures d'attribution d'un permis de séjour (asile, visa, regroupement familial, etc.). De plus, différents acteurs privés (avocat·e.s), associatifs et militants se saisissent du droit pour en négocier l'application 18 .

Ainsi, les associations sont impliquées de différentes manières dans la mise en œuvre et la production des politiques migratoires ${ }^{19}$, de sorte que celle-ci se fait «avec, et en partie par, les associations de la cause des étrangers » 20 . Le recours au droit des associations se révèle ambivalent ${ }^{21}$, permettant de résister "par le bas " aux pratiques de l'État ${ }^{22}$, tout en reproduisant en partie ses catégories de traitement à travers la sélection des dossiers 23 et l'approche visant à faire cadrer toute requête avec le droit 24 .

Dans ce dossier, nous poursuivons donc les travaux sur l'application du droit de la migration - qui réglemente les questions d'accès au territoire, au séjour et à la protection internationale - en analysant différents acteurs " de terrain " (agent.e.s de l'administration, magistrat.e.s, interprètes, avocat.e.s, salarié.e·s des associations ou militant.e.s) - comme des intermédiaires du droit qui contestent, mettent en œuvre, mais aussi contribuent à la production du droit de la migration et à sa redéfinition. La notion d'intermédiation est relativement récente et a d'abord été utilisée pour étudier le processus et les acteurs qui contribuent à l'endogénéité du droit 25 , en particulier dans le champ de la régulation des activités économiques et

16. Julia DAHLVIK, «Why Handling Power Responsibly Matters: The Active Interpreter Through the Sociological Lens ", in Nick GILL et Anthony Good (eds.), Asylum Determination in Europe: Ethnographic Perspectives, Cham : Springer International Publishing, 2019, p. 133-154; Robert GIBB et Anthony GooD, « Interpretation, Translation and Intercultural Communication in Refugee Status Determination Procedures in the UK and France », Language and Intercultural Communication, 14 (3), 2014, p. 385-399.

17. Federica Infantino, Outsourcing Border Control: Politics and Practice of Contracted Visa Policy in Morocco, New York : Palgrave Macmillan, 2016.

18. Nicolas FISCHER, «Une frontière "négociée”. L'assistance juridique associative aux étrangers placés en rétention administrative", Politix, 87, 2009, p. 71-92.

19. Estelle D'Halluin-Mabillot, Les épreuves de l'asile. Associations et réfugiés face aux politiques du soupçon, Paris : Éditions de l’École des Hautes Études en Sciences Sociales, 2012.

20. Mathilde PETTE, «Associations : les nouveaux guichets de l'immigration? Du travail militant en préfecture», Sociologie, 5 (4), 2014, p. 405-421.

21. Liora ISRAËL, "Faire émerger le droit des étrangers en le contestant, ou l'histoire paradoxale des premières années du GISTI ", Politix, 62, 2003.

22. Laura ODASSO, «L'action des groupes militants en faveur des couples binationaux. Entre soutien humain, juridique et conscientisation », Recherches familiales, 14, 2017, p. 119-133.

23. Jonathan MIAZ, «Mobiliser le droit pour défendre les réfugiés : les ambivalences des guichets juridiques ", in Martina Avanza, Jonathan Miaz, Cécile PÉCHu et Bernard Voutat (dir.), Militantismes de guichet. Perspectives ethnographiques, Lausanne : Antipodes, 2021 (à paraître).

24. Estelle D'HALluin, «Passeurs d'histoire. L'inconfort des acteurs associatifs impliqués dans l'aide à la procédure d'asile ", in Didier FASSIN (dir.), Les nouvelles frontières de la société française, Paris : La Découverte, 2010, p. 363-383. Laura ODASSO et Manuela SALCEDO ROBLEDO, «Intimacy Brokers. The Fragile Boundaries of Activism for Heterosexual and Same-Sex Binational Couples in France », in Anne-Marie D'Aoust, Transnational Marriage and Partner Migration: Constellations of Security, Citizenship, and Rights, New Brunswick: Rutgers University Press, 2021 (à paraître).

25. Lauren B. EDELMAN, «L'endogénéité du droit », in Christian BESSY, Thierry DelPeuCH et Jérôme PÉLISSE (dir.), Droit et régulations des activités économiques : perspectives sociologiques et institutionnalistes, Paris :LGDJ Lextenso éditions, 2011, p. 85-110. 
du travail 26 . Les intermédiaires du droit désignent alors les acteurs qui manient et mobilisent des règles juridiques dans leurs activités professionnelles, militantes ou associatives ${ }^{27}$. Dans ce dossier, nous suivons la proposition de Jérôme Pélisse de ne pas limiter l'étude des intermédiaires du droit aux seul.e.s professionnel.le·s du droit (juges, avocat·e.s, conseils juridiques). Il propose ainsi d'élargir l'analyse à toute une variété d'acteurs, y compris des non-professionnel-le·s du droit, des street-level bureaucrats et d'autres frontline workers $^{28}$ pour saisir la manière dont ils et elles traduisent le droit des livres (law-in-books) en droit en actes (law-in-action).

Une analyse en termes d'intermédiaires du droit permet, ici, d'opérer deux mouvements par rapport à la littérature existante sur les politiques migratoires. Premièrement, elle permet de mettre l'accent sur les manières dont ces différent.e.s professionnel-le·s mobilisent et travaillent les règles juridiques pour mettre en œuvre ou contester les politiques migratoires. Deuxièmement, cette perspective d'analyse permet de «tenir ensemble » ces différent·e.s acteur-trice-s et de saisir la manière complexe dont leurs usages du droit interagissent les uns avec les autres pour, in fine, produire le droit de la migration. Ainsi, par rapport aux travaux sur les street-level bureaucrats, parler d'intermédiaires du droit et d'intermédiation juridique conduit à porter le regard sur les dimensions processuelles 29 et relationnelles de la mise en œuvre du droit de la migration et de sa production " par le bas » en incluant dans l'analyse une palette de professionnel-le.s plus large que les seul.e.s street-level bureaucrats (par exemple des acteurs privés, tel.le·s que les avocat.e.s, ou encore des acteurs associatifs et militants qui mobilisent le droit). Dès lors, le questionnement transversal de ce dossier peut être formulé comme suit : comment est-ce que différent.e.s intermédiaires du droit contribuent, en relation et collectivement, d'une part, à la mise en œuvre ou à la contestation du droit de la migration et, d'autre part, à la production de politiques migratoires orientées vers la fermeture des frontières ou, au contraire, à une politique d'accueil plus favorable aux migrant.e.s?

Les différents articles ici rassemblés accordent donc une place particulière aux interactions entre intermédiaires du droit et à la dimension relationnelle du travail d'intermédiation. Dans leur pratique du droit d'asile, les juges assesseurs de la Cour nationale du droit d'asile (CNDA) interagissent avec différents acteurs - associatifs, militants, experts, administratifs, judiciaires - qui, avec les caractéristiques propres

26. Jérôme PÉLISSE, «Travailler le droit : lectures et perspectives sociologiques », Revue française de sociologie, 59 (1), 2018, p. 99.

27. Les publications suivantes présentent un panorama très complet des travaux sur les intermédiaires du droit et de l'approche en termes d'intermédiation du droit: Sebastian BILlows, Lisa Buchter et Jérôme PÉLISSE, « Introduction: The Microfoundations of Legal Intermediation in Organizational Contexts », Studies in Law, Politics, and Society, 81, 2019, p. 1-9 ; Garry GraY et Jérôme PÉLISSE, « Frontline Workers and the Role of Legal and Regulatory Intermediaries", Sciences Po LIEPP Working Paper, 94, 2019, p. 1-17 ; Shauhin TALESH et Jérôme PÉLISSE, « How Legal Intermediaries Facilitate or Inhibit Social Change », Studies in Law, Politics and Society, 79, 2019, p. 111-145.

28. Jérôme PÉLISSE, "Varieties of Legal Intermediaries: When Non-Legal Professionals Act as Legal Intermediaries ", Studies in Law, Politics and Society, 81, 2019, p. 101-128.

29. Sebastian Billows, Lisa Buchter et Jérôme PÉLISSE, «Introduction: The Microfoundations of Legal Intermediation in Organizational Contexts ", op. cit. 
aux juges - leurs trajectoires, dispositions et éthos professionnel -, influencent la manière dont les juges assesseurs traduisent la convention de Genève (Romane Sabrié et Jean-Luc Richard). Dans cette procédure judiciaire auprès de la CNDA, les interprètes exercent un "contrôle" sur les récits des demandeur.se-s d'asile en leur rendant ou non la parole à l'issue de leur traduction d'une portion du récit (Christian Licoppe et Maud Verdier). Ces acteur-trice-s sont donc des intermédiaires du droit dans la mesure où ils et elles interviennent, en interaction avec d'autres intermédiaires (juges et avocat.e.s) et avec les demandeur.se-s d'asile, dans la co-construction des « états de faits " sur lesquels les juges doivent statuer.

L'analyse de la judiciarisation de la politique d'asile en Suisse met en exergue comment la production du droit d'asile se poursuit, au-delà de l'arène parlementaire, sur le terrain de la mise en œuvre du droit à travers les interactions conflictuelles entre les pratiques de l'administration et celles de la défense juridique des migrant.e.s (Jonathan Miaz). Les controverses juridiques autour du « délit de solidarité » à la frontière franco-italienne montrent quant à elles comment les avocat.e.s et les inculpé·e.s questionnent les frontières entre ce qui est (il)légal et ce qui est (il)légitime, et comment leurs collaborations dans le cadre de procès font émerger différentes conceptions, parfois antagonistes, de l'engagement de solidarité en faveur des migrant.e.s. Non seulement ces relations entre des intermédiaires du droit et leurs client.e.s configurent la manière dont les premiers mobilisent le droit et la justice pour défendre les seconds et, plus généralement, leur cause, mais ces relations peuvent aussi susciter des tensions dans l'identité professionnelle des avocat.e.s (Annalisa Lendaro). Enfin, la judiciarisation massive du contentieux des étrangers permet de relever l'interdépendance entre des intermédiaires ayant des représentations extrêmement variables de leur travail au nom du droit de la migration. Les interactions entre les avocat.e.s, les juristes et les travailleurs et travailleuses associatifs, les magistrat.e.s administratif.ve.s et les assistant.e.s de justice, ainsi que leurs représentations réciproques - sources des clivages entre et au sein de ces groupes professionnels - montrent le visage humain du droit au recours (Laura Odasso).

\section{Spécificités du droit de la migration}

Adoptant une telle approche, il convient de mentionner quelques traits caractéristiques du droit de la migration qui le distinguent des autres domaines juridiques analysés jusqu'à présent. Tout d'abord, ce droit est traversé par des tensions entre les questions de souveraineté, auxquelles renvoie la gestion de l'immigration, et celles des droits fondamentaux ${ }^{30}$, auxquelles renvoie l'adhésion aux conventions internationales et au droit européen. De plus, ce domaine du droit fait l'objet d'une politisation constante d'ordre sécuritaire ${ }^{31}$, voire xénophobe. Les politiques migratoires, notamment dans le domaine de l'asile, sont aussi fortement judiciarisées ${ }^{32}$,

30. Marie-Laure BASILIEN-GAINCHE, « Sécurité des frontières et/ou protection des droits ", Cités, 46 (2), 2011, p. 47-68.

31. JefHuYSMAns, The Politics of Insecurity: Fear, Migration, and Asylum in the EU, Londres : Routledge, 2006.

32. Rebecca Hamlin, Let Me Be a Refugee: Administrative Justice and the Politics of Asylum in the United States, Canada, and Australia, New York: Oxford University Press, 2014 ; Leila KAWAR, Contesting Immigration 
ce qui conduit à une interdépendance forte entre les différents acteurs impliqués dans ces procédures (agents administratifs, corps judiciaire, associations actives dans l'aide juridique, avocat.e.s). En cela, le droit de la migration constitue un exemple édifiant d'une judiciarisation de l'action publique. Caractérisé par un infradroit 33 florissant, le droit de la migration place les migrant.e.s dans des situations d'insécurité juridique - instrument par excellence des régimes migratoires ${ }^{34}$ - marquées par l'exclusion 35 et le contrôle ${ }^{36}$. Ce droit implique donc une importante dépossession et, par extension, " une remise de soi » 37 des migrant.e.s, souvent illégalisé·e·s et placé·e·s dans une situation de précarité économique et existentielle, caractérisée par la perte d'emprise sur leurs propres vies, ainsi que par les menaces d'arrestation et de renvoi forcé ${ }^{38}$. En outre, ce droit, dont l'application passe par la catégorisation de situations complexes et hybrides, laisse aux acteurs des dispositifs d'immigration une importante marge d'interprétation des trajectoires et des requêtes des étranger.e.s 39 .

Du fait de l'ambiguïté des règles juridiques, l'action des intermédiaires du droit, capables de les interpréter et de socialiser les individus à son usage, a pris une ampleur grandissante au fil de l'évolution des politiques migratoires 40 et des changements de

Policy in Court: Legal Activism and its Radiating Effects in the United States and France, New York: Cambridge University Press, 2015 ; Leila KAWAR et Jonathan MIAZ, "Enacting Immigration Politics in a Juridical Register ", in Shauhin TALesh, Heinz Klug et Elizabeth MerTz (eds.), Research Handbook on Modern Legal Realism, Northampton : Edward Elgar Publishing, 2021, p. 161-175 ; Dagmar SOENNECKEN, « The Managerialization of Refugee Determinations in Canada ", Droit et Société, 84, 2013, p. 291-311.

33. Danièle LoСНAK, Étrangers, de quel droit?, Paris : PUF, coll. « Politique d'aujourd'hui », 1985.

34. Catherine DAUvergne, Making People Illegal. What Globalization Means for Migration and Law, Cambridge : Cambridge University Press, 2008 ; Lionel GALLIANO, « La situation juridique de l'étranger en France ou l'insécurité comme moyen de régulation de l'immigration », Asylon(s), 4, 2008 ; Alexis SPIRE, "La politique des guichets au service de la police des étrangers », Savoir/Agir, 36 (2), 2016, p. 27-31.

35. François CREPEAU, «Les réfugiés : l'exclusion par le droit ", in Lucie LEMONDE (dir.), Droit et droits : de

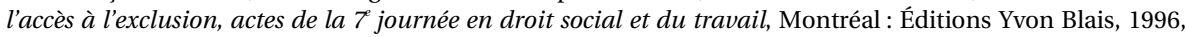
p. 107-121.

36. Ségolène Barbou des Places, «La catégorie en droit des étrangers : une technique au service d'une politique de contrôle des étrangers", Asylon(s), 4, 2008 ; Gallya LAHAV et Virginie GUIRAUDON, "Actors and Venues in Immigration Control: Closing the Gap between Political Demands and Policy Outcomes », West European Politics, 29 (2), 2006, p. 201-223.

37. Nous reprenons la notion de remise de soi à propos de la représentation juridique, notamment, par analogie avec l'usage que Pierre Bourdieu fait de cette notion (qu'il désigne aussi par celle de fides implicita) à propos de la représentation politique. Dans le contexte de la représentation juridique, la notion de remise de soi désigne la délégation globale et totale par laquelle les migrant·e.s s'en remettent aux intermédiaires du droit pour leur procédure, leur accordant leur confiance, tout en étant dépossédé.e.s du contrôle sur la procédure et les démarches entreprises. Pierre BOURDIEU, « La représentation politique. Éléments pour une théorie du champ politique ", Actes de la recherche en sciences sociales, 36-37, 1981, p. 3-24 ; Céline BESSIÈRE, Muriel Mille et Gabrielle ScHüTZ, «Les avocat.es en droit de la famille face à leur clientèle. Variations sociales dans la normalisation de la vie privée », Sociologie du travail, 62 (3), 2019 ; Vincent-Arnaud CHAPPE, "La qualification juridique est-elle soluble dans le militantisme? Tensions et paradoxes au sein de la permanence juridique d'une association antiraciste », Droit et Société, 76, 2010.

38. Giada DE Coulon, L'illégalité régulière: ethnographie du régime de l'aide d'urgence en Suisse, Lausanne : Antipodes, 2019.

39. Cécile Rousseau, François CréPEAu, Patricia FoXEN et France Houle, « The Complexity of Determining Refugeehood: A Multidisciplinary Analysis of the Decision-Making Process of the Canadian Immigration and Refugee Board », Journal of Refugee Studies, 15 (1), 2002, p. 43-70.

40. Nicolas FIsCHER et Camille HAMIDI, Les politiques migratoires, Paris : La Découverte, 2016. 
l'État. Ces acteurs facilitent les procédures d'immigration et participent au tri migratoire en reproduisant des conceptions du "mérite ", de la "véracité », de la "crédibilité » et de «l'intégration " produites par l'État et les discours politiques, voire en introduisant des indicateurs d'appréciation nouveaux sur la base de leur travail quotidien. Interroger la position de ces intermédiaires dans les dispositifs d'immigration nationaux et leurs rôles complexes aide à éclairer les dynamiques de traduction, de transmission, d'usages, voire de résistance et de désobéissance au droit dans les démarches administratives, au-delà et en-deçà de celles-ci.

\section{Un regard sur les intermédiaires du droit}

À la lumière de ce constat, les contributions réunies dans ce dossier thématique resserrent la focale d'analyse sur les "intermédiaires du droit» qui configurent l'accès au séjour en France et en Suisse. Des méthodologies d'enquête variées fondent les recherches empiriques à la source de ce dossier, qui se caractérise par la mise en lien des dynamiques d'usages du droit propres à plusieurs domaines du droit de la migration (protection internationale, droit au recours, protection des mineurs) ainsi que par une diversité de procédures - administratives et judiciaires - et d'intermédiaires du droit (fonctionnaires, juges assesseurs, interprètes, avocat.e.s, travailleurs et travailleuses sociaux, bénévoles et juristes associatifs, militant·e.s).

Romane Sabrié et Jean-Luc Richard s'intéressent aux juges assesseurs de la Cour nationale du droit d'asile (CNDA) en France afin de comprendre qui juge l'asile. L'article présente la pratique du droit au sein de cette juridiction comme le produit d'interactions entre différents mondes et expertises administrative, associative et militante ou judiciaire. La variété des approches et donc des trajectoires de chacun des juges assesseurs dicte la co-construction d'un éthos professionnel. Le partage de cet éthos professionnel renvoie l'assesseur à sa posture de décideur et de garant du droit d'asile En traduisant la convention de Genève, en mobilisant les articles juridiques qui la régissent, l'assesseur devient un intermédiaire de ce droit, de son application en contexte français et qui participe à sa création. En effet, les incertitudes propres aux contentieux sont source de jurisprudence qui, elle, fait de la CNDA une instance créatrice de droit.

Dans le même contexte, Christian Licoppe et Maud Verdier éclairent le travail des interprètes impliqués dans les audiences de la CNDA. Les questions de prises de parole analysées avec des méthodes multimodales renvoient à une microsociologie des relations de pouvoir qui impliquent les juges, les demandeur.se-s d'asile et intègrent les interprètes. Cet article montre ainsi que, lorsque les interprètes terminent leur traduction d'une partie d'une réponse longue apportée par un·e requérant.e d'asile à une question d'un.e juge, ils et elles peuvent alors soit rendre la parole pour que la personne puisse poursuivre sa réponse, soit la laisser au juge pour qu'il ou elle pose une autre question. Les interprètes, par leur activité verbale et gestuelle agissent ainsi sur les possibilités des requérant.e-s d'asile de faire entendre leur voix (ou non) devant la Cour. Cet article met aussi en évidence comment ces processus d'attribution de la parole par les interprètes sont sensibles à 
l'écologie des tribunaux, en particulier si l'interprète se trouve physiquement à côté du ou de la demandeur.se d'asile ou non.

Jonathan Miaz aborde ensuite la judiciarisation de la politique suisse d'asile en s'intéressant aux interactions entre les associations qui défendent juridiquement les migrant.e.s et l'administration en charge d'instruire les demandes d'asile en Suisse. Cette étude de cas met en évidence une dynamique particulière de la judiciarisation de la politique d'asile, basée sur la contestation massive de décisions individuelles (dimension quantitative) et sur une jurisprudence foisonnante aux effets significatifs sur les pratiques des acteurs de terrain (dimension qualitative). J. Miaz montre ainsi comment les usages stratégiques du droit par l'administration et par les acteurs de la défense juridique des migrant.e.s - analysés comme autant d'intermédiaires du droit - se répondent et comment ce processus de judiciarisation se poursuit dans les réactions du Parlement et du Gouvernement aux jurisprudences du Tribunal administratif fédéral, témoignant du caractère circulaire de l'action publique. En définitive, l'auteur met en évidence la co-production conflictuelle du droit d'asile.

Dans le contexte de la judiciarisation de la solidarité envers les migrant·e·s, c'est-à-dire ici de procès contre des personnes qui ont apporté leur aide à des migrant·e.s à la frontière franco-italienne, Annalisa Lendaro éclaire les relations de connivence et d'interdépendance entre les personnes inculpées, qui se définissent comme "solidaires" ou "militant·e·s", et les avocat·e·s engagé·e·s qui les défendent. Cet article analyse tout particulièrement les tensions entre ces cause lawyers et les « délinquant·e·s solidaires " autour de la manière dont ils et elles conçoivent la cause, ainsi que les stratégies judiciaires à adopter et leur dimension politique. Cette contribution d'Annalisa Lendaro montre notamment comment le travail d'intermédiation juridique peut faire entrer en tension l'éthos professionnel des juristes avec la défense d'une cause, ce qui a des conséquences sur la manière dont ils et elles vont mobiliser le droit pour défendre leurs client·e·s, élaborer des stratégies judiciaires et investir l'arène judiciaire en restant fidèle à leur éthos professionnel impliquant le respect de l'État de droit ou en recourant à une défense plus politique.

Enfin, Laura Odasso explore les différentes significations que des avocat.e.s, des travailleurs et des travailleuses associatifs, des magistrat.e.s administratif.ve.s et des assistant.e.s de justice, relié.e.s les un.e.s aux autres dans le cadre du contentieux en droit des étrangers, donnent à leur pratique juridique. L'analyse de leur éthos professionnel permet de dégager quatre types de significations qui définissent leur aire et choix d'action. La première considère le travail en matière de droit des étrangers comme "peu noble "; la deuxième motive sa pratique selon la «trajectoire familiale et personnelle » du ou de la professionnel-le; la troisième la qualifie en fonction d'une croyance dans la "primauté de la déontologie du métier d'avocat.e " et la quatrième d'un "militantisme légal et politique». Ce travail de signification met également en exergue les clivages qui traversent ces groupes professionnels et leurs représentations réciproques. Par un jeu d'interaction et de positionnements en opposition et en dialogue avec les autres, ces professionnel-le·s définissent leur posture en tant qu'intermédiaires de la migration et de sa judiciarisation. Par cette 
analyse compréhensive, la contribution de cette autrice a montré, plus largement, que les trajectoires biographiques des intermédiaires et leurs visions du droit (voire des droits) - c'est-à-dire comment ils pensent le droit et son rôle dans la société, et comment ils l'interprètent et l'utilisent - façonnent l'application de la loi.

En définitive, ces contributions portent non seulement sur les usages du droit et les différents ressorts de la " force du droit » 41 , mais aussi sur les pratiques et les représentations sociales déterminant les manières dont le droit est mobilisé, utilisé et contesté au quotidien par des acteurs intermédiaires qui agissent en interaction et qui produisent des formes spécifiques d'incorporation du droit de la part des étranger·e.s.

\section{- Les auteur'rice's}

Jonathan Miaz est chercheur au Centre de droit comparé européen et international de l'Université de Lausanne. Ses recherches portent sur la mise en œuvre des politiques migratoires à l'aune des pratiques des administrations, des tribunaux et des mobilisations. Il travaille actuellement sur les usages politiques du droit international dans les cantons suisses. Il a notamment publié :

— «Entre examen individuel et gestion collective : automatisation des décisions dans la procédure d'asile en Suisse? ", Lien social et Politiques, 83, 2019;

— «Le Droit et ses médiations. Pratiques d'instruction des demandes d'asile et encadrement institutionnel des décisions ", Politique et Sociétés, 38 (1), 2019.

Laura Odasso est chercheure à la chaire Migrations et Sociétés du Collège de France et fellow de l'Institut Convergences Migrations. Ses recherches explorent l(es)'expérience(s) et les usages que les familles mixtes et migrantes font du droit et de l'action publique migratoire, ainsi que le rôle joué par le monde associatif et les professionnels du droit qu'ils rencontrent dans leur parcours. Elle a notamment publié :

— « Negotiating Legitimacy: Binational Couples in the Face of Immigration Bureaucracy in Belgium and Italy ", Anthropologica (Journal), 63 (1), 2021 ;

- Faire et défaire les liens familiaux. Usages et pratiques du droit en contexte migratoire (avec Aurélie FILlOD-CHABAUD), Rennes : Presses universitaires de Rennes, 2020.

Romane Sabrié est doctorante en sociologie à l'Université Paris Nanterre (laboratoire Sophiapol, ED EOS) et affiliée à l'Institut Convergences Migrations. Elle travaille actuellement à une sociohistoire de l'accueil des demandeurs d'asile en France et notamment de la genèse du Dispositif national d'accueil par l'histoire de France terre d'asile.

41. Pierre Bourdieu, « La force du droit », Actes de la recherche en sciences sociales, 64 (1), 1986, p. 3-19. 